\title{
The Requirement Analysis of Software based on Use Case Modeling
}

\author{
Changhong SONG \\ Beijing Institute of Petrochemical Technology, Beijing 102617, China
}

\begin{abstract}
Use case modeling is an important component of object-oriented software development technology, which can perfectly capture system functional requirements, reflect the mutual relationship between the user and the system. Methods using the use case modeling completed the requirement analysis of student achievement management system.
\end{abstract}

KEYWORD: Requirement analysis; Use case modeling; Actor

\section{INTRODUCTION}

Software requirement analysis is an important step in software life cycle, which is the most critical step. Only through software requirement analysis, analyst can to describe the overall concept of software functions and performance for a specific software requirements specification, and the establishment of software development. Requirement analysis is the first stage of software project, it is the task of determining what the system must do, so as to meet the requirements of users, therefore need a way to accurately the needs of users of copolymer [1]. Use case modeling is a kind of help us accurately grasp the needs of the user method, it has good performance, can let users understand the function of the system, allows developers to accurately grasp the implementation of system [2]. Analyst needed careful understanding of user requirements, detailed investigation and analysis, to convert the user requirements into a complete and detailed software model.

\section{USE CASE MODEL}

Use case model is used to describe the specified system all use cases, actors and use cases participant relationship combination [3]. In UML, the use case model is described by a plurality of use case diagram and the use case specifications.

\subsection{Use case diagram}

The main elements of the use case diagram is participants (Actor), case (Use Case) and the relationship between them, which represents all the use cases and interaction of participants in a system.

\subsection{Actor}

Use case modeling method firstly describes defined system which participants, the participants and the defined system interact, can be a person, a computer system or some other runnable process.

\subsection{Use case}

For each participant, use case modeling method to describe the system provides what kind of services for the actors (i.e., use case), which defines how a system used by actors. It is a system function of the externally visible, these functions are provided by the system, and through the exchange of messages between with the actors to express. A use case is one of the core concepts of the unified modeling language UML. A use case is a typical interaction between the user and the computer.

The following use case features:

1) Use cases capture some visible user needs, to achieve a specific user goals;

2) Use cases are activated by the executive, and provide the exact value for the executive;

3) Use cases can be big or small, but it must be for a specific user goals integrity description of the.

\subsection{Relationship}

In the use case diagram, there are a variety of relationships between use cases and actors, and between actors. Used to indicate the relationship of 
actors which use service system, and connects the actors and use cases, the relationship between them is called a communication link. Among the actors can exist generalization, said actors can be enumerated sub actor. At the same time, can also use case between inclusion, extension and generalization relationship.

Inclusion relation (include): when there is a large piece of similar action exists in a few cases, and do not want to repeat the action can be used to describe, described the case contains a simple other use case behavior, the use of uses in the old version.

Extended relational (extend): when a case similar to another case, but doaction can use some special situation, describes the extended use case to the basic case, the use of extends in the old version.

Generalization relationship: case can be listed as the number of sub cases.

\subsection{Use case specification}

Just describe the system from the use case diagram is not enough, with information describing each case also need, namely the use case specification. The use case specifications basically is the text mode is used to describe, it mainly describes the business process. The basic components include the following.

Use case name: names of all cases that readers should stick out a mile to know the purpose of a use case, and is the only.

Brief description: a brief description of the role, purpose of use case.

The stream of events: text description of the behavior of the system from the use case point. Stakeholders should be able to understand these descriptions, generally by the basic flow and alternative flow and sub stream constitutes. Sometimes also includes the visualization of the flow of events.

Pre conditions: system constraints defined case start.

Post conditions: system constraints defined cases after the termination of the,

Relations: some relationship cases participation, such as a communication link.

In order to more clearly describe the flow of events, the use case specifications can also choose to use the state diagram, activity diagram and sequence diagram to illustrate. As long as the concise expression of use cases help, you can add other graphics in a use case. [4]

\section{APPLICATION IN STUDENT ACHIEVEMENT MANAGEMENT SYSTEM}

Student achievement management system needs to meet the demand from three aspects, these three aspects are the educational administrators, teachers and students. The needs of the students is the query himself achievement, credit information; educational administrator users all kinds of information on student achievement and student information and course information for global operations; to carry on the statistical analysis of results; teacher user functional requirements granted granted query course grades, course results entry, analysis course grades. The teacher can input student achievement, once submitted after the results cannot be modified.

The student result management module to complete the user through the query results, students households according to my student ID and password to login the system, users can only query term students their test scores, the total score. Access to personal credit inquiry for me. Add password not only satisfy the student user requirements, but also to protect the student user privacy.

Educational administrators have realized all the student achievement management by the achievement management module. The main function modules of student achievement information according to the class of query, modify, statistics and other functions. Educational administrator for the course information, student information, teacher information, grade information, class information, credit's information can execute the query, modify operation. The whole system is the core of educational administrator, user's security level will be very high. Educational administrators can also for each user has permission to modify the password.

Teacher user has input subjects taught student achievement and student performance statistics operation. Teacher user can $\log$ on to the system according to my teacher's ID and password. Teachers can only input yourself taught course student achievement information; curriculum teaches classes the query student achievement, score, average score, pass rate, excellent rates, scores query. The amount of information the teacher user functions, data security and confidentiality requirements of the highest, as show in figure 1 .

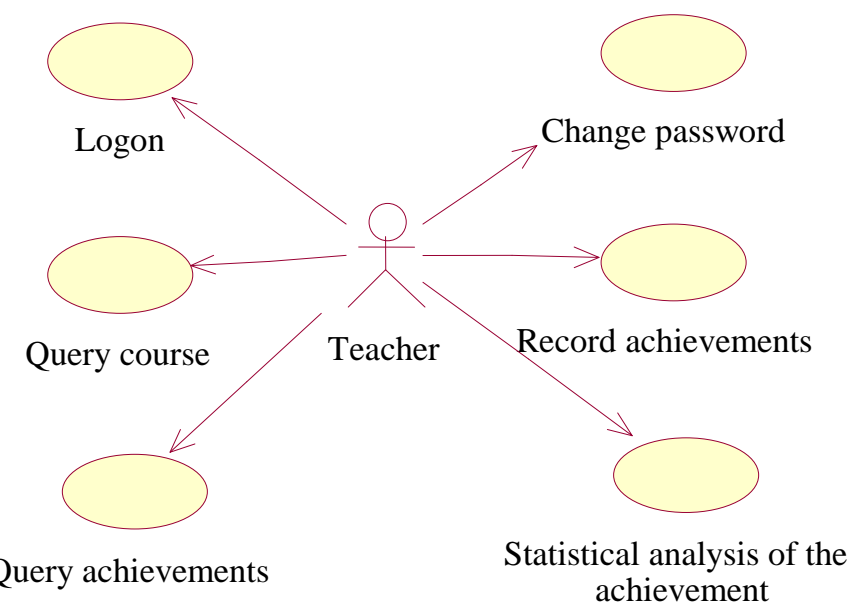

Figure 1. Use case figure of teacher user 
This system, for each case are given to the use case description, due to limited space, only giving the use case of teacher user query achievements, as shown in table 1 .

Table 1. Use case specification

\begin{tabular}{|l|l|}
\hline Use case name & Query achievements \\
\hline actor & Teacher user \\
\hline Pre conditions & Teacher user already logged in the course grade already exists \\
\hline post condition & Query performance information \\
\hline & 1.Tteachers into the query class student achievement information interface; \\
The main stream of events & 3.Tthe teacher input class number; \\
& 4.The teacher Input the date of the examination \\
& 5.Displays performance information; \\
\hline
\end{tabular}

\section{CONCLUSION}

Functional requirements use case modeling are clearly defines the specific tasks performed by participants, the user can clearly see the system provides valuable operation. Software requirement modeling use case modeling technology, can effectively reduce the complexity of the system under development, help people to understand complex issues [5]. Need to note is that the list of design characteristics of case does not care about the system design, interface or testing, it is just a requirements document. Use case of the capture is functional requirements of the system (or called system behavior), and the system nonfunctional requirements, we need to obtain the demand model of the other, or use supplementary specification to describe. When using the use case modeling, we must understand the stakeholders and user's groups, determine the boundary of participants and system; to understand the participant is not always people; if the system boundary definition is not clear, it will cause a vague definition of system function.

\section{REFERENCES}

[1] Wang Feng, Shi Bingxin, Luo Li. Research of UML modeling mechanism and application in requirement analysis of system. Computer Engineering and Design, 2005, 26 (4): 971 975.

[2] Zhu Weiping, Tan Hansong. Discussion on the application of use case technology. Journal of XiangNan University, 2005, 26 (2): 66 70.

[3] Stev e Ado LPH Paul Bramble. Effective use cases model. EPress.CN Che $\mathrm{Li}$ red. Beijing: Tsinghua University press, 2003.

[4] Zhang Yi, Application of use case modeling in the requirement analysis. Science and technology information 2010, 36:12 13.

[5] Yan Jianen, Wang Cuihua, Lin Jianqiu, Wang Junyi. Use case modelingin software requirement analysis. Journal of Inner Mongolia University (NATURAL SCIENCE EDITION), 2007.9, 38 (5):578 581. 